\title{
Removing Characteristic of Indoor Air Pollutants According to Activated Carbon Pot and Plant Using a Tin Oxide Gas Sensor
}

\author{
Takashi OYABU, ${ }^{\text {a* }}$ Ayako SAWADA, ${ }^{\text {b }}$ Tomoaki HASHIMOTO, ${ }^{c}$ and Takayuki YOSHIOKA \\ ${ }^{a}$ Graduate School of Regional Economic Systems, Kanazawa Seiryo University (Gosho-machi 10, Kanazawa, Ishikawa \\ 920-8620, Japan) \\ ${ }^{\mathrm{b} S}$ School of Knowledge Science, Japan Advanced Institute of Science and Technology (Asahidai 1-1, Tatsunokuchi, \\ Ishikawa 923-1211, Japan) \\ cTokyo Metropolitan Agricultural Experiment Station (Fujimi-machi 3-8-1, Tachikawa, Tokyo 190-0013, Japan)
}

Received June 18, 2004 ; Accepted September 14, 2004

\begin{abstract}
Activated carbon has a high porous structure and it can absorb many kinds of chemical substances in an atmosphere. In this study, the capability was examined for formaldehyde, toluene and xylene using a tin oxide gas sensor, which are named as VOCs(volatile organic compounds). An interior plant pot was made of the activated carbon and was adopted as a subject. It had a high capability to remove those chemicals. The pot, which was installed in an experimental chamber, could absorb entirely the formaldehyde of about $6.5 \mathrm{ppm}$ in five hours. It also had a removing capability to larger molecular weight chemicals, for example toluene and xylene. The sensor has higher sensitivity to the gases because there are a lot of free radicals on the sensor surface and many free electrons generate in the sensor bulk. Based on these results, the three pots were installed in a real office room and examined the removing capability. In the room, the formaldehyde was emitted continuously and the saturated concentration was almost constant. The concentration was decreased until $60 \%$ by installing the pots. They had a high removing capability to continuously emitting formaldehyde. The sensor output was sometimes calibrated by a formaldehyde measuring instrument. In the experimental room, temperature, humidity, light intensity, oxygen and carbon dioxide concentrations were also measured simultaneously. The characteristics had a correlation each other.
\end{abstract}

Key Words : V()C. Indoor Air Pollution, Activated Carbon. Plant, Iabor Environment

\section{Introduction}

Various types of synthetic chemicals have been accumulated in an atmosphere according to economic growth. These chemicals are existing in an indoor environment and harm humans. These chemicals create serious indoor air quality issues. The domicile and commercial building have been adopted a tightly sealed structure due to an energy saving measure. As a result, the rate of persons who become a sick building syndrome, has been increasing. And the phenomenon becomes one of social issues. Namely the work productivity decreases and medical cost due to their diseases increases.

It is necessary to decrease the concentration of air pollutants. The best method for decreasing these chemicals in an indoor environment is the ventilating. It is, however, an unreasonable manner in night-time and in winter months. The ventilation using a fan is in need of energy. In the meantime, it is good to adopt an optical catalyst, for example titanium oxide to decrease the pollutants and it is also effective to use the activated carbon. Plants have the purification capability to decompose their chemicals. ${ }^{1-6)}$ The optical catalyst is restricted to act under a light. The activated carbon has the capability to absorb the chemicals temporarily, for example toluene, xylene and formaldehyde, but the capability saturates some day. It is, however, useful to remove the high concentrating air-pollutants.
In this study, the activated carbon was adopted to examine its purification capability in an indoor environment. It is made of palm husks. The goal of this study is to manufacture the activated carbon pot growing an interior plant. Plants can remove airborne chemicals continuously. The pot with plant has a capability to reduce a high concentrating pollution and decompose the pollutants continuously by the plant. It has a synergism effect for absorbing and decomposing the substances. It can sustain cleanly the indoor environment, for instance labor environment and residential space. We hope that this type of pot will spread widely to reduce the diseases concerning with sick building syndrome.

\section{Regulations for Chemicals in an Indoor Environment}

Sick house syndrome has become one of serious issues. It is caused by existing various kinds of chemicals in an atmosphere, for example formaldehyde, toluene and xylene which are mainly emitted from the building materials. In Japan, the revised Building Standard Law was introduced to restrict the use of formaldehyde in 2003. The incidence of sick house syndrome will be reduced by the law. It has a capability to reduce the total volume of air polluting chemicals. It is strongly desired to comply with the law. The influences of formaldehyde to humans are summarized in Table 1 . The threshold 
Table 1 The influences to human body due to formaldehyde concentrations.

\begin{tabular}{l|c}
\multicolumn{1}{c|}{ Influence } & Concentration(ppm) \\
\hline Threshold value for smell & 0.08 \\
\hline The stimulus value to an eye & 0.4 \\
\hline The inflammation value of a throat & 0.5 \\
\hline The stimulus to a nose and an eye & 2.6 \\
\hline Lachrymal & 4.6 \\
\hline
\end{tabular}

value of odor sense of formaldehyde is $0.08 \mathrm{ppm}$ and its value is also adopted as a guideline of Ministry of Health, Labor and Welfare in Japan. Toluene is restricted to 0.07 $\mathrm{ppm}$ and xylene is to $0.20 \mathrm{ppm}$ in the living and labor environments. It is not permitted that the concentration of toluene reaches $50 \mathrm{ppm}$ temporarily and xylene reaches $100 \mathrm{ppm}$ in work environment.

\section{Absorption of Air Pollutants onto Activated Carbon}

SEM image of activated carbon using in this experiment is shown in Fig. 1. A plant pot was made of the carbon. The carbon was made of palm husks. It has a porous structure and specific surface is about $1000 \mathrm{~m}^{2} / \mathrm{g}$. It can absorb various kinds of chemicals. The plant pot was manufactured using the activated carbon. The diameter of the pot is $23 \mathrm{~cm}$, the height is also $23 \mathrm{~cm}$ and the weight is $1.3 \mathrm{~kg} \pm 0.065 \mathrm{~kg}$. The manufactured activated carbon pot is shown in Fig. 2.

The pot may absorb many kinds of air pollutants, for example toluene and xylene. The absorbing capabilities

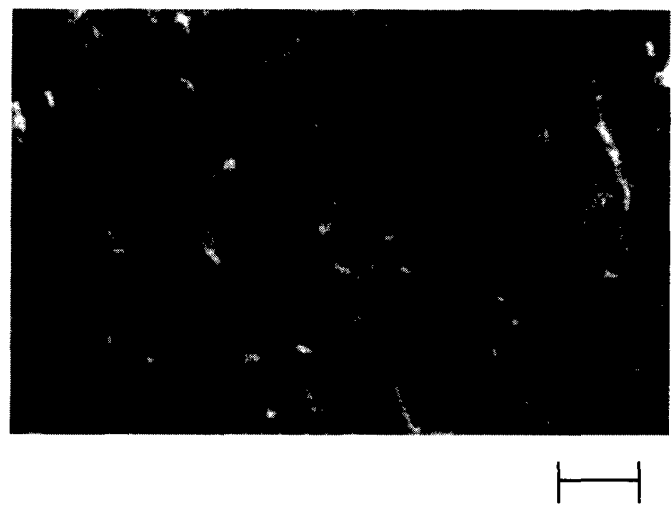

$10 \mu \mathrm{m}$

Fig. 1 SEM image of activated carbon manufactured from palm shell.

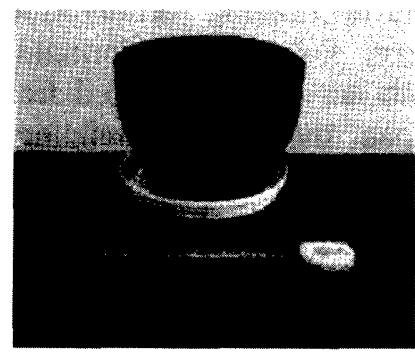

Fig. 2 Photograph of activated carbon pot. of the pot were examined in an experimental chamber of 300 litters. The capabilities are shown in Fig. 3. In the figure, the vertical axis means the concentration grade of each pollutant and horizontal axis means the passage time. The concentration grade was measured using a tin oxide gas sensor (Figaro, TGS\#826). ' The photograph of the sensor is shown in Fig. 4. The sensor output becomes larger as the concentration becomes higher. When the characteristic decreases quickly, the pot has higher purification capability.

The molecular weights of toluene and xylene are larger than that of formaldehyde. It takes long time to decompose those molecular-weight pollutants using plants. Plants have high purification capabilities to various kinds of air pollutants in an atmosphere. The capability, however, becomes lower as the molecular weight of substance becomes larger. ${ }^{2}$ The pot has higher absorbing effect to the chemicals. It is desired strongly to remove the pollutants in an indoor environment.

The absorbing characteristic of the carbon pot for formaldehyde was examined. It is indicated in Fig. 5. Formaldehyde was injected into the chamber using a micro syringe and the concentration in the chamber became at $6.5 \mathrm{ppm}$. After injection, formaldehyde diffuses in the chamber and the concentration become uniform promptly. The sensor output, which indicates the concentration, increases immediately at the injection. The output reaches the peak value and decreases gradually according to the absorption of the chemical onto the activated carbon surface. Finally, it returns to the original level which is called "base level". The height from the

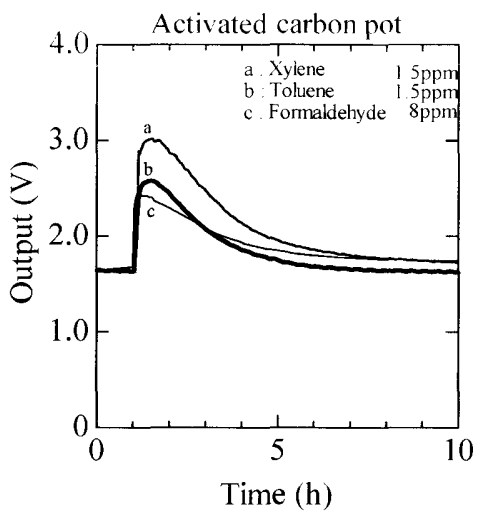

Fig. 3 The adsorbing capabilities of activated carbon pot for formaldehyde, toluene and xylene.

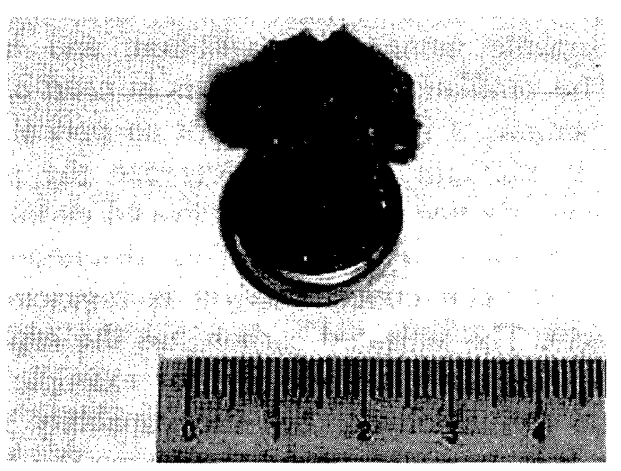

Fig. 4 Photograph of the adopted gas sensor. 
base level to peak level is named as $h$. The full-width at a half maximum of the characteristic is tagged as $t_{w}$. The absorption capability $P_{a}$ is derived using $h$ and $t_{w}$. It is shown in equation (1). In Fig. 5, the derived $P_{a}$ was 32.4 and $P_{a}$ of the control was 2.7. There was a small amount of leakage volume in the chamber. The results for Figs. 3 and 5 are also summarized in Table 2. The absorption capability of the pot becomes greater as the molecular weight of the pollutant becomes larger. It is considered that the number of free electrons in the sensor bulk will increase in the case of higher molecular-weight pollutants because the number of free radical will increase by the catalytic reaction on the sensor surface. Therefore $P_{a}$ s for toluene and xylene become larger than the one for formaldehyde. This phenomenon is related to sensorsensitivity to the chemical. The pot is very effective to reduce the chemicals in the human environment, especially it can remove the larger molecular weight substances and it is principally effective in highly contaminated environment like a newly built house.

$$
P_{a}=h / t_{w} \times 100
$$

\section{Absorption Effect of Activated Carbon Pots in an Office Room}

The absorption capability of the activated carbon pot for formaldehyde was measured in a real office room. The photograph of the room in which the three pots are placed, is shown in Fig. 6 and the layout of the room is also shown in Fig. 7. The capacity of the room is about $47.5 \mathrm{~m}^{3}$ and five persons can work in the room. The experiments were carried out in a warm season. In this experiment, there was no person in the room. There is a window whose width is about $4.8 \mathrm{~m}$ and it faces east. The room was left lights off. The window height is $60 \mathrm{~cm}$.

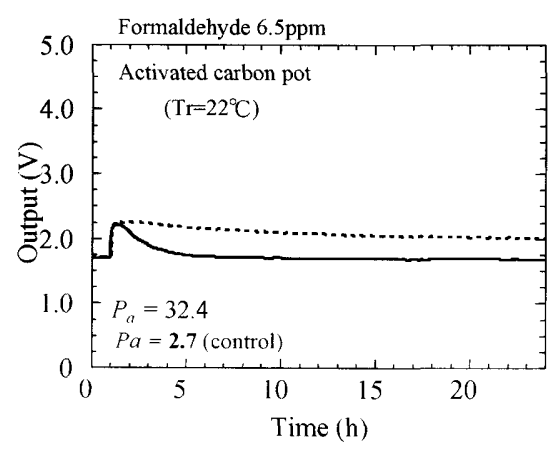

Fig. 5 The sensor output characteristics for the injection of formaldehyde and its control. The atmosphere temperature was 22 degree $\mathrm{C}$.

Table 2 The absorption characteristics by the activated carbon pot for formaldehyde, toluene and xylene. $h$ means peak value, $t_{w}$ means the full-width at half maximum and $P_{a}$ is the removing capability.

\begin{tabular}{cccc}
\hline & HCHO 6.5 ppm & Toluene $1.5 \mathrm{ppm}$ & Xylene $1.5 \mathrm{ppm}$ \\
\hline$h$ & 0.52 & 0.95 & 1.38 \\
$t_{w}$ & 1.6 & 1.92 & 2.42 \\
$P_{a}$ & 32.4 & 49.4 & 57.1 \\
\hline
\end{tabular}

The air conditioner always operated and room temperature was about 24 to 26 degree C. Formaldehyde generating container was placed at right side of Fig. 7 .

The absorption characteristic of formaldehyde onto the surface of activated carbon pot in the room was examined using a tin oxide gas sensor (New Cosmos Electric, $\mathrm{CH}-\mathrm{N}$ ). The formaldehyde concentration was input into a mobile computer through an A-D converter every minute and the correct concentration was also detected intermittently using the measuring instrument of formaldehyde (Riken, FP-85). These data were checked each other. The measuring instrument is an optical type and uses a test tablet every experiment. Light intensity, temperature, humidity, carbon dioxide and oxygen concentrations were also detected as well as formaldehyde concentration.

The characteristics of six sensors (temperature \& humidity: T\&D Co., TR-72S, oxygen: New Cosmos, HS-60, carbon dioxide: New Cosmos, IRC-630, light intensity: T $\&$ D Co., PHR-51) are shown in Fig. 8. After 24 hour from the beginning of the experiment, formaldehyde was generated from the container in the room and the concentration increased as shown in Fig. 8 (a). The three activated carbon pots were placed 48 hours later. Since the time, the gas-sensor output decreased gradually, namely the formaldehyde concentration decreased. Whole sensor-characteristics were measured for 6 days. The formaldehyde measuring device which was the tablet type, was also used intermittently to identify the concentration precisely. The concentration at 48 hour was $1.6 \mathrm{ppm}$ and

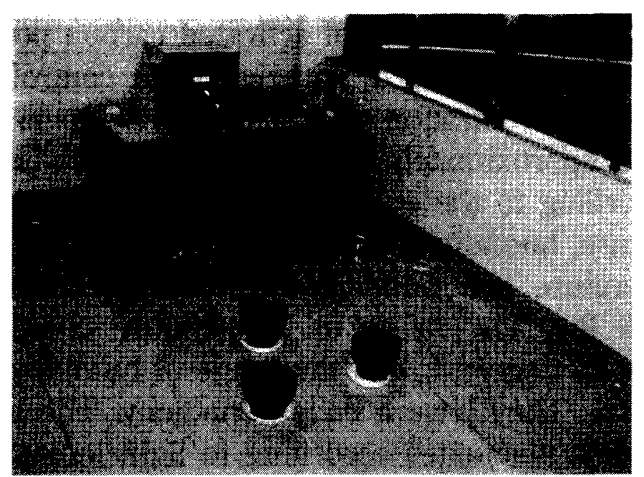

Fig. 6 Photograph of the room in which the experiment was carried out.

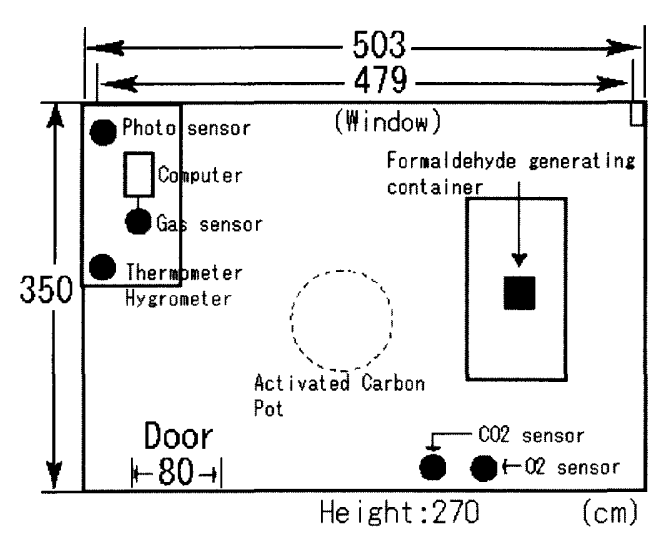

Fig. 7 The layout of the room. 


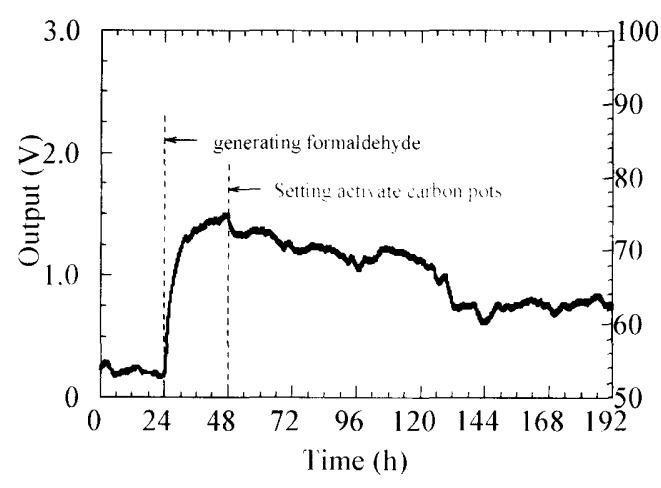

(a) gas sensor output

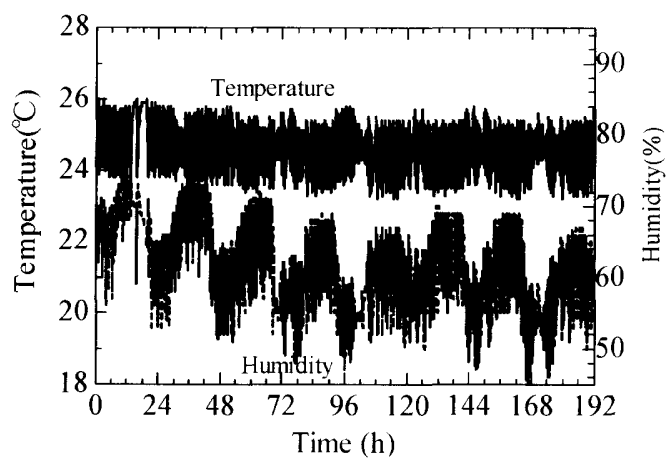

(b) temperature and humidity

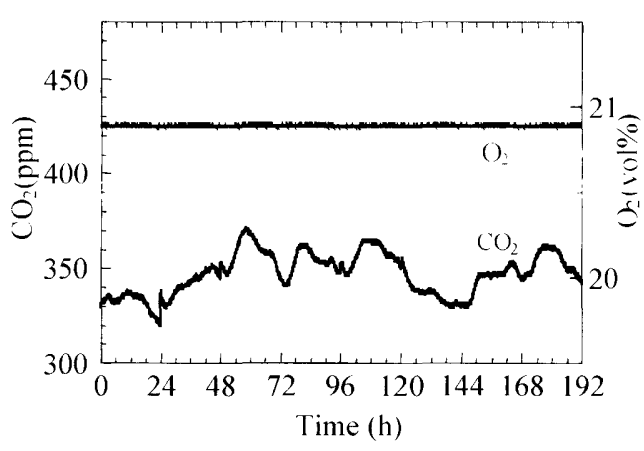

(c) oxygen and carbon dioxide

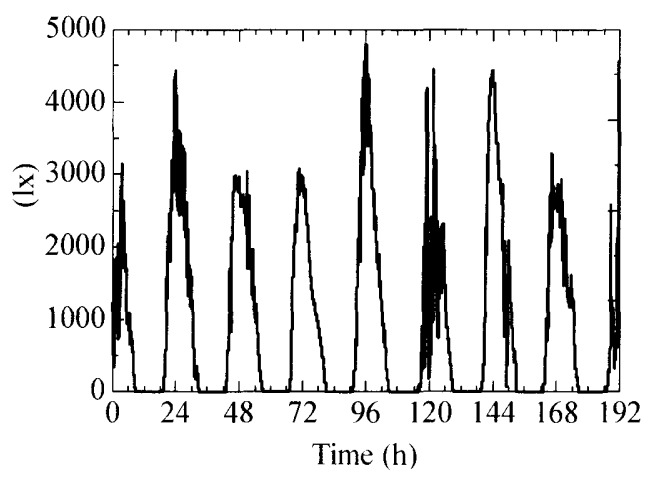

(d) light intensity

Fig. 8 Six sensor characteristics when formaldehyde was continuously generated and it was absorbed onto the surfaces of three carbon pots.

$1.0 \mathrm{ppm}$ at 192 hour. Therefore, the formaldehyde was absorbed onto the surface of activated carbon pot. When the pots were not placed, namely in the control experiment, the concentration characteristic from 48 to 192 hours was approximately constant but it somewhat fluctuated according to the environmental factors for instance, temperature, humidity and light intensity.

Temperature and humidity characteristics are shown in Fig. 8 (b). The range of temperature characteristic was 24 to 26 degree $\mathrm{C}$ and the range of humidity was 50 to $70 \mathrm{RH} \%$. They have a daily periodicity. The humidity characteristic fluctuates according to light intensity from the outside and temperature. The room was always controlled by an air conditioner. The oxygen and carbon dioxide characteristics are also shown in Fig. 8 (c). There was no person in the room, so the concentration of carbon dioxide was 330 to $370 \mathrm{ppm}$ and the concentration of oxygen was almost $20.9 \%$. The light intensity characteristic is shown in Fig. 8 (d). It takes maximum value in daytime and almost zero in night-time. There is a negative correlation between humidity and light intensity characteristics, and positive correlation among temperature and light intensity characteristics. Temperature and humidity characteristics changes according to light intensity.

\section{Removing Capability of Activated Carbon Pot with Plant}

The activated carbon pot has a high absorption capability to higher molecular weight chemicals such as xy-

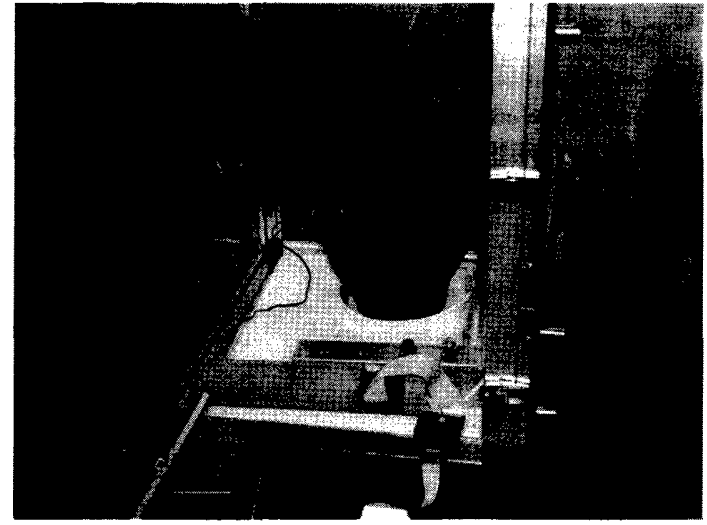

Fig. 9 Photograph of experimental chamber which contains the geranium pot.

lene and toluene. So it can be thought that the activated carbon pot with a plant will have a higher removing capability to various kinds of air pollutants. It is also thought that there is a limit in the absorption volume of the pot without a plant. It is necessary to derive both of a plant purification capability and the absorption capability of the activated carbon. It is acceptable for the pot to have a continuously removing capability. The removing capability of the activated carbon pot with a plant as a subject was investigated. The photograph for the subjective pot is shown in Fig. 9. The geranium was growing in the pot and it was placed in the experimental chamber of 300 litters. There was the tin oxide gas sensor (Figaro, TGS 826 ) in front of the pot in the chamber. The experimen- 


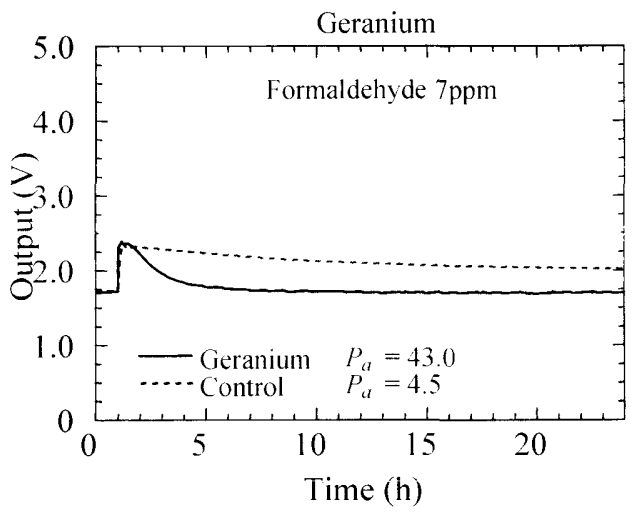

Fig. 10 The removing characteristic of geranium pot.

tal system is the same as the one used in Fig. 3.

The removing characteristic is indicated in Fig. 10. In the figure, formaldehyde was injected into the chamber 1 hour later from the beginning of the experiment. The concentration is $7 \mathrm{ppm}$ which is about 100 times as much as the regulation concentration. The purification capability $P_{a}$ of the characteristic is 43 , and that of control is 4.5. The capability of pot is 10 times higher than the control. The value is almost same as the pot without the plant because the surface area of the pot decreases by planting the geranium. The inside of the pot is covered with the soil so the removing capability of the pot decreases.

The removing characteristic of the pot with a plant for xylene of $1.5 \mathrm{ppm}$ is shown in Fig. 11. $P_{a}$ was 52.3 and the value was higher. It is almost same with the data of Table 2. When the same sized plant was growing in a plastics pot, the $P_{a}$ was about 7 . Therefore, the activated carbon pot had higher removing capability. It is excellent. The removing capability according to the pot is higher than the one for the plant.

The removing characteristic of the pot with the peace lily for toluene of $1.5 \mathrm{ppm}$ is shown in Fig. 12. The figure resembles in the characteristic of Fig. 11. The activated carbon pot has also higher removing characteristic to $\mathrm{xy}$ lene and toluene.

\section{Conclusion}

The removing capabilities of the activated carbon pots without and with a plant to the important air pollutants were investigated. The activated carbon is made of the palm husks. Firstly, the experiments were carried out in the chamber of 300 litters and the capability was evaluated using the proposed equation. The value was derived using the tin oxide sensor output characteristic. The pot had the high removing capability to the larger molecularweight substances. It is effective in a newly built house. The experiments were also carried out in the real office room. Formaldehyde was emitted continuously in the room, and it was examined that the activated carbon pot was useful to remove the chemical. The characteristic was investigated using a gas sensor. As for the results, the pots could remove the continuously emitting formaldehyde, namely the concentration decreases from 1.6 to

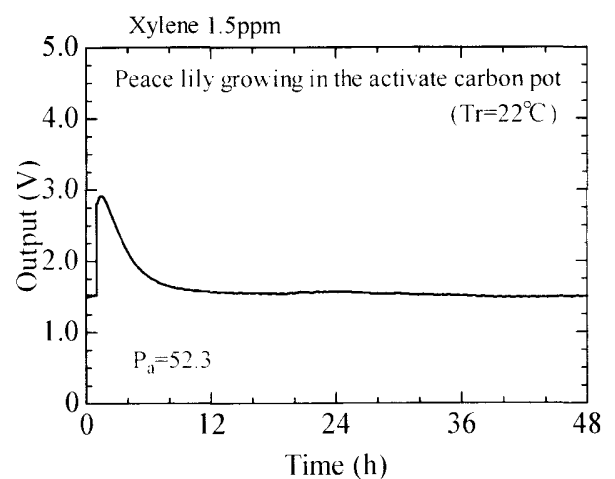

Fig. 11 The removing characteristic of the activated carbon pot for xylene of $1.5 \mathrm{ppm}$, in which a peace lily is growing.

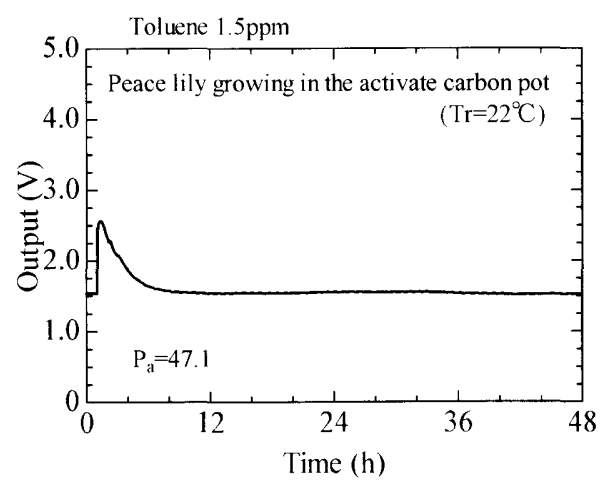

Fig. 12 The toluene removing characteristic of the activated carbon pot for toluene of $1.5 \mathrm{ppm}$, in which a peace lily is growing.

$1.0 \mathrm{ppm}$. The capability of the pot with the plant was also measured. It also indicated a high removing capability. The plants have higher removing, capabilities to low molecular weight substances. The activated carbon pot with a plant has a synergism for removing both lower and higher molecular weight substances. The capability of the pot may be influenced with temperature and humidity.

Labor environment will be improved by applying these results to a commercial building. The productivity of the workers will also increase.

\section{References}

1) K. Omasa, H. Saji, S. Youssefian, and N. Kondo, Air Pollution and Plant Biotechnology, Springer, Tokyo (2002).

2) T. Oyabu, T. Onodera, H. Kimura, and Y. Sadaoka, J. Jpn. Soc. Atmos. Environ., 34, 319 (2001).

3) T. Oyabu, A. Sawada, T. Onodera, K. Takenaka, and Bill Wolverton, Sens. Actuators B, 89, 131 (2003).

4) T. Oyabu, A. Sawada, T. Onodera, and K. Takenaka, Electrochemistry, 71, 463 (2003).

5) T. Oyabu, K. Takenaka, Bill Wolverton, T. Onodera, and H. Nanto, Internat. J. Phytoremed., 5, 267 (2003).

6) A. Sawada, T. Oyabu, and T. Yoshida, ITE Lett., 4, 673 (2003).

7) Figaro General Catalogue, Figaro TGS Gas Sensor, p.7, Osaka, Japan (1983). 